e-print JLAB-THY-97-25

June 1997

\title{
Asymmetric Parton Distributions
}

\author{
ANATOLY V. RADYUShKin \\ Physics Department, Old Dominion University, Norfolk, VA 23529, USA \\ Jefferson Lab, Newport News, VA 23606, USA
}

\begin{abstract}
Applications of perturbative QCD to hard exclusive electroproduction processes in the Bjorken limit at small invariant momentum transfer $t$ bring in a new type of parton distributions which have hybrid properties, resembling both the parton distribution functions $f_{a}(x)$ and the distribution amplitudes. Their $t$-dependence is analogous to that of hadronic form factors. We discuss general properties of these new parton distributions, their relation to usual parton densities and the evolution equations which they satisfy.
\end{abstract}

\section{INTRODUCTION}

The use of phenomenological functions accumulating information about nonperturbative long-distance dynamics is the standard feature of applications of pQCD to hard processes. The well-known examples are the parton distribution functions $f_{p / H}(x)$ [1] used in $\mathrm{pQCD}$ approaches to hard inclusive processes and distribution amplitudes $\varphi_{\pi}(x)$ [2] etc. which naturally emerge in the asymptotic QCD analyses of hard exclusive processes. Recently, it was argued that the same gluon density $f_{g}(x)$ used for description of hard inclusive processes also determines the amplitudes of hard exclusive $J / \psi$ [3] and $\rho$-meson [4] electroproduction. It was also proposed [5] to use another exclusive process of deeply virtual Compton scattering $\gamma^{*} p \rightarrow \gamma p^{\prime}$ (DVCS) for measuring parton distribution functions inaccessible in inclusive measurements. An important feature (noticed long ago [6 8]) is that kinematics of DVCS and hard elastic electroproduction processes, in a frame where the hadron moves fast, requires the presence of the longitudinal component in the momentum transfer $r \equiv p-p^{\prime}$ from the initial hadron to the final: $r_{\|}=\zeta p$. This means that the matrix element $\left\langle p^{\prime}|\ldots| p\right\rangle$ is essentially asymmetric ("skewed"). For DVCS and $\rho$-electroproduction in the region $Q^{2}>>|t|, m_{H}^{2}$, the "skewedness" parameter $\zeta$ coincides with the Bjorken variable $x_{B j}=Q^{2} / 2(p q)$. For diffractive processes, both the skewedness $\zeta$ of the nonperturbative matrix element $\left\langle p^{\prime}|\ldots| p\right\rangle$ and the absolute value of the momentum transfer $t \equiv\left(p^{\prime}-p\right)^{2}$ are small. Studying the DVCS process, one should be able to consider the whole region $0 \leq \zeta \leq 1$ and $t \sim 1 G^{2}$ [9]. In this situation, one deals with essentially nonforward (or off-forward in terminology of ref. [5]) matrix element $\left\langle p^{\prime}|\ldots| p\right\rangle$. The pQCD approaches incorporating asymmetric/off-forward parton distributions were formulated in refs. 5 5.92 12]. A detailed analysis of pQCD factorization for hard meson electroproduction processes was given in ref. [13. The basic idea of our papers [10,11] is that one should treat the initial momentum $p$ and the longitudinal part of the momentum transfer $r$ on equal footing by introducing double distributions $F(x, y)$, which specify the fractions of $p$ and $r_{\|}$carried by the active parton: $k_{\|}=x p+y r_{\|}$. These distributions look like distribution functions with respect to $x$ and like distribution amplitudes with respect to $y$. The longitudinal momentum transfer $r_{\|}$is proportional to $p: r_{\|}=\zeta p$ and it is convenient to parametrize matrix elements $\langle p-r|\ldots| p\rangle$ by nonforward $\mathcal{F}_{\zeta}(X ; t)$ or, in the $t \rightarrow 0$ limit, asymmetric distribution functions $\mathcal{F}_{\zeta}(X)$ specifying the total light-cone fractions $X p=(x+y \zeta) p,(X-\zeta) p \equiv X^{\prime} p$ of the initial hadron momentum $p$ carried by the "outgoing"

*Also at Laboratory of Theoretical Physics, JINR, Dubna, Russia 
and "returning" partons on the skewedness parameter $\zeta$ while the asymmetric distribution functions $\mathcal{F}_{\zeta}(X)$ form a family of $X$-dependent functions changing their shape when $\zeta$ is changed The functions $\mathcal{F}_{\zeta}(X)$ also have hybrid properties. When $X \geq \zeta$, the returning parton carries a positive fraction $(X-\zeta) p$ of the initial momentum $p$, and $\mathcal{F}_{\zeta}(X)$ is similar to the usual parton distribution $f(X)$. In the region $0 \leq X \leq \zeta$ the difference $X-\zeta$ is negative, i.e., the second parton should be treated as propagating together with the first one. The partons in this case share the longitudinal momentum transfer $r_{\|}=\zeta p$ in fractions $Y \equiv X / \zeta$ and $1-Y$ : in the region $X \leq \zeta$ the function $\mathcal{F}_{\zeta}(X)$ looks like a distribution amplitude.

It is very important to know spectral properties of the parton distributions $F(x, y)$ and $\mathcal{F}_{\zeta}(X)$. In ref. 12 , it was shown that both arguments of a double distribution $F(x, y)$ satisfy the natural "parton" constraints $0 \leq x \leq 1$, $0 \leq y \leq 1$ for any Feynman diagram contributing to $F(x, y)$. A less obvious restriction $0 \leq x+y \leq 1$ 10 12 ] guarantees that the argument $X=x+y \zeta$ of the asymmetric distribution $\mathcal{F}_{\zeta}(X)$ also changes between 0 and 1 . Since $X=0$ can be obtained only if both $x=0$ and $y=0$, due to vanishing phase space for such a configuration the asymmetric distributions $\mathcal{F}_{\zeta}(X)$ vanish for $X=0$. This property is very essential, because the hard subprocess amplitudes usually contain $1 / X$ factors. When $\mathcal{F}_{\zeta}(0) \neq 0$, one faces a non-integrable singularity $\mathcal{F}_{\zeta}(X) / X$ at $X=0$.

\section{ASYMMETRIC DISTRIBUTIONS}

There are two leading-twist quark operators $\bar{\psi}_{a}(0) \gamma_{\mu} E(0, z ; A) \psi_{a}(z)$ and $\bar{\psi}_{a}(0) \gamma_{5} \gamma_{\mu} E(0, z ; A) \psi_{a}(z)$, where $E(0, z ; A)$ is the usual path-ordered exponential which makes the operators gauge-invariant. In the forward case, the first one gives the spin-averaged densities $f_{a}(x)$ while the second one is related to the spin-dependent ones $\Delta f_{a}(x)$. Here, we will discuss the $\bar{\psi}_{a} \gamma_{\mu} E(0, z ; A) \psi_{a}$ operators and gluonic operators with which they mix under evolution. The relevant nonforward matrix element can be written as

$$
\begin{aligned}
& \left.\left\langle p^{\prime}, s^{\prime}\left|\bar{\psi}_{a}(0) \hat{z} E(0, z ; A) \psi_{a}(z)\right| p, s\right\rangle\right|_{z^{2}=0} \\
& =\bar{u}\left(p^{\prime}, s^{\prime}\right) \hat{z} u(p, s) \int_{0}^{1}\left(e^{-i X(p z)} \mathcal{F}_{\zeta}^{a}(X ; t)-e^{i(X-\zeta)(p z)} \mathcal{F}_{\zeta}^{\bar{a}}(X ; t)\right) d X \\
& +\bar{u}\left(p^{\prime}, s^{\prime}\right) \frac{\hat{z} \hat{r}-\hat{r} \hat{z}}{2 M} u(p, s) \int_{0}^{1}\left(e^{-i X(p z)} \mathcal{K}_{\zeta}^{a}(X ; t)-e^{i(X-\zeta)(p z)} \mathcal{K}_{\zeta}^{\bar{a}}(X ; t)\right) d X,
\end{aligned}
$$

where $\hat{z} \equiv z^{\mu} \gamma_{\mu}$. In Eq.(11), quark and antiquark contributions are explicitly separated (cf. [15]). As emphasized by $\mathrm{X}$. Ji [5], the parametrization of this nonforward matrix element must include both the nonflip functions $\mathcal{F}_{\zeta}(X ; t)$ and the spin-flip functions which we denote by $\mathcal{K}_{\zeta}(X ; t)$. Taking the $O(z)$ term of the Taylor expansion gives the sum rules [5]

$$
\begin{array}{r}
\int_{0}^{1}\left[\mathcal{F}_{\zeta}^{a}(X ; t)-\mathcal{F}_{\zeta}^{\bar{a}}(X ; t)\right] d X=F_{1}^{a}(t) \\
\int_{0}^{1}\left[\mathcal{K}_{\zeta}^{a}(X ; t)-\mathcal{K}_{\zeta}^{\bar{a}}(X ; t)\right] d X=F_{2}^{a}(t)
\end{array}
$$

relating the nonforward distributions $\mathcal{F}_{\zeta}^{a}(X ; t), \mathcal{K}_{\zeta}^{a}(X ; t)$ to the $a$-flavor components of the Dirac and Pauli form factors, respectively. For gluons, the nonforward distribution can be defined through the matrix element

$$
\begin{aligned}
& \left.\left\langle p^{\prime}\left|z_{\mu} z_{\nu} G_{\mu \alpha}^{a}(0) E^{a b}(0, z ; A) G_{\alpha \nu}^{b}(z)\right| p\right\rangle\right|_{z^{2}=0} \\
& =\bar{u}\left(p^{\prime}\right) \hat{z} u(p)(z \cdot p) \int_{0}^{1} \frac{1}{2}\left[e^{-i X(p z)}+e^{i(X-\zeta)(p z)}\right] \mathcal{F}_{\zeta}^{g}(X ; t) d X+{ }^{~ " \mathcal{K}}{ }_{\zeta}^{g "} .
\end{aligned}
$$

In the formal $t=0$ limit, the nonforward distributions $\mathcal{F}_{\zeta}(X ; t), \mathcal{K}_{\zeta}(X ; t)$ convert into the asymmetric distribution functions $\mathcal{F}_{\zeta}(X), \mathcal{K}_{\zeta}(X)$. In the $\zeta=0$ limit, $\mathcal{F}_{\zeta}(X)$ reduce to the usual parton densities $\mathcal{F}_{\zeta=0}^{a}(X)=$ $f_{a}(X) ; \mathcal{F}_{\zeta=0}^{g}(X)=X f_{g}(X)$. For small $\zeta, \mathcal{F}_{\zeta}^{a}(X)$ differs from $f_{a}(X)$ by $O(\zeta)$

\footnotetext{
${ }^{\dagger}$ Asymmetric distribution functions defined in ref. [11] are similar to, but not coinciding with the $t \rightarrow 0$ limit of the off-forward parton distributions introduced by X.Ji [5].

${ }^{\ddagger}$ A more detailed discussion of double distributions and their relation to nonforward distributions was given in my talk about DVCS 14.
} 


\section{EVOLUTION EQUATIONS}

Near the light cone $z^{2} \sim 0$, the bilocal operators $\bar{\psi}(0) \ldots \psi(z)$ develop logarithmic singularities $\ln z^{2}$, and the limit $z^{2} \rightarrow 0$ is singular. Calculationally, these singularities manifest themselves as ultraviolet divergences for the light-cone operators. The divergences are removed by a subtraction prescription characterized by some scale $\mu$ : $\mathcal{F}_{\zeta}(X ; t) \rightarrow \mathcal{F}_{\zeta}(X ; t ; \mu)$. In QCD, the gluonic operator $\mathcal{O}_{g}(u z, v z)=z_{\mu} z_{\nu} G_{\mu \alpha}^{a}(u z) E^{a b}(u z, v z ; A) G_{\alpha \nu}^{b}(v z)$ mixes under renormalization with the flavor-singlet quark operator. In the leading logarithm approximation, the easiest way to get the evolution equations for nonforward distributions is to use evolution equations [16.17 for light-ray operators:

$$
\mu \frac{d}{d \mu} \mathcal{O}_{a}(0, z)=\int_{0}^{1} \int_{0}^{1} \sum_{b} B_{a b}(u, v) \mathcal{O}_{b}(u z, \bar{v} z) \theta(u+v \leq 1) d u d v
$$

where $\bar{v} \equiv 1-v$ and $a, b=g, Q$. For valence distributions, there is no mixing, and their evolution is generated by the $Q Q$-kernel alone. Inserting Eq.(6) between chosen hadronic states and parametrizing matrix elements by appropriate distributions, one can get DGLAP and BL-type kernels and also to calculate the new kernels $R^{a b}(x, y ; \xi, \eta)$ and $W_{\zeta}^{a b}(X, Z)$. The kernels $R^{a b}(x, y ; \xi, \eta)$ govern the evolution of double distributions:

$$
\mu \frac{d}{d \mu} F^{a}(x, y ; t ; \mu)=\int_{0}^{1} d \xi \int_{0}^{1-\xi} \sum_{b} R^{a b}(x, y ; \xi, \eta) F^{b}(\xi, \eta ; t ; \mu) d \eta .
$$

The form of equations is not affected by the $t$-dependence, and " $t$ " will not be indicated in what follows. Since integration over $y$ converts $F^{a}(x, y)$ into the parton density $f_{a}(x)$, whose evolution is governed by the DGLAP equation, the kernel $R_{Q Q}(x, y ; \xi, \eta)$ has the property

$$
\int_{0}^{1-x} R_{Q Q}(x, y ; \xi, \eta) d y=\frac{1}{\xi} P_{Q Q}(x / \xi) .
$$

Integrating $R_{Q Q}(x, y ; \xi, \eta)$ over $x$ one gets the BL-type kernel:

$$
\int_{0}^{1-y} R_{Q Q}(x, y ; \xi, \eta) d x=V_{Q Q}(y, \eta) .
$$

Another set of kernels $W_{\zeta}^{a b}(X, Z)$ describes the evolution of nonforward distributions and asymmetric distribution functions:

$$
\mu \frac{d}{d \mu} \mathcal{F}_{\zeta}^{a}(X ; t ; \mu)=\int_{0}^{1} \sum_{b} W_{\zeta}^{a b}(X, Z) \mathcal{F}_{\zeta}^{b}(Z ; t ; \mu) d Z
$$

When $\zeta=1$, the initial momentum coincides with the momentum transfer and $\mathcal{F}_{\zeta}(X)$ reduces to a distribution amplitude whose evolution is governed by the BL-type kernels: $W_{\zeta=1}^{a b}(X, Z)=V^{a b}(X, Z)$. The BL-type kernels appear as a part of the asymmetric kernel $W_{\zeta}^{a b}(X, Z)$ even in the general $\zeta \neq 1,0$ case [12]. As explained earlier, if $X$ is in the region $X \leq \zeta$, then the function $\mathcal{F}_{\zeta}(X)$ can be treated as a distribution amplitude $\Psi_{\zeta}(Y)$ with $Y=X / \zeta$. For this reason, when both $X$ and $Z$ are smaller than $\zeta$, the kernels $W_{\zeta}^{a b}(X, Z)$ simply reduce to the BL-type evolution kernels $V^{a b}(X / \zeta, Z / \zeta)$. Furthermore, the BL-type kernels also govern the evolution in the region corresponding to transitions from a fraction $Z$ which is larger than $\zeta$ to a fraction $X$ which is smaller than $\zeta$. Note, that the evolution jump through the critical fraction $\zeta$ is irreversible: $X \leq \zeta$ if $Z \leq \zeta$. To put it in words, when the parton momentum degrades in the evolution process to values smaller than the momentum transfer $\zeta p \equiv r$, further evolution is like that for a distribution amplitude: the momentum can decrease or increase up to the $r$-value but cannot exceed this value.

When $X>\zeta$, the initial quark momentum $X p$ is larger than the momentum transfer $r=\zeta p$, and the asymmetric distribution function $\mathcal{F}_{\zeta}^{a}(X)$ is a generalization of the usual distribution function $f_{a}(X)$ for a skewed kinematics. Hence, the evolution in the region $\zeta<X \leq 1, \zeta<Z \leq 1$ is similar to that generated by the DGLAP equation. The relevant kernels $P_{\zeta}^{a b}(X, Z)$ can be obtained from the light-ray kernels, e.g.,

$$
P_{\zeta}^{Q Q}(X, Z)=\frac{Z-X}{Z Z^{\prime}} \int_{0}^{1} B_{a b}\left(\bar{w}(1-X / Z), w\left(1-X^{\prime} / Z^{\prime}\right)\right) d w
$$

where $Z^{\prime} \equiv Z-\zeta$. Note, that $P_{\zeta}^{Q Q}(X, Z)$ is given by a function symmetric with respect to the interchange of $X, Z$ with $X^{\prime}, Z^{\prime}$. In the $\zeta \rightarrow 0$ limit, $P_{\zeta}^{Q Q}(X, Z)$ converts into the DGLAP kernel: $P_{\zeta=0}^{Q Q}(X, Z)=P^{Q Q}(X / Z) / Z$. 
Acknowledgement. This work was supported by the US Department of Energy under contract DE-AC05-84ER40150.

[1] R.P.Feynman, The Photon-Hadron Interactions, W.A.Benjamin,Inc., 1972.

[2] A.V. Efremov and A.V. Radyushkin, Theor. Math. Phys. 42 (1980) 97.

[3] M.G. Ryskin, Z. Phys. C37 (1993) 89.

[4] S.J. Brodsky et al. Phys.Rev. D50 (1994) 3134.

[5] X. Ji, Phys.Rev.Lett. 78 (1997) 610.

[6] J. Bartels and M. Loewe, Z.Phys. C12 (1982) 263.

[7] L.V. Gribov, E.M. Levin and M.G. Ryskin, Phys.Reports, 100 (1983) 1.

[8] F.-M. Dittes et al. Fortschr.Phys. 42 (1994) 101.

[9] X. Ji, Phys.Rev. D55 (1997) 7114; hep-ph/9609381.

[10] A.V. Radyushkin, Phys. Lett. B380 (1996) 417.

[11] A.V. Radyushkin, Phys. Lett. B385 (1996) 333.

[12] A.V. Radyushkin, preprint JLAB-THY-97-10; hep-ph/9704207.

[13] J.C. Collins, L. Frankfurt, and M. Strikman, preprint CERN-TH/96-314.

[14] A.V. Radyushkin, these Proceedings.

[15] A.V. Efremov and A.V. Radyushkin, Lett. Nuovo Cim. 19 (1977) 83.

[16] Th. Braunschweig et al. Ann. Phys. (Leipzig) 44 (1987) 403.

[17] I.I. Balitsky and V.M. Braun, Nucl.Phys. B311 (1988/89) 541. 